\title{
Promoter methylation changes and vascular dysfunction in pre-eclamptic umbilical vein
}

\author{
Qinqin Gao ${ }^{1 *}$, Xiaorong Fan ${ }^{1 \dagger}$, Ting $\mathrm{Xu}^{1 \dagger}$, Huan $\mathrm{Li}^{1}$, Yun He${ }^{1}$, Yuxian Yang ${ }^{4}$, Jie Chen ${ }^{1}$, Hongmei Ding ${ }^{1}$, \\ Jianying $\mathrm{TaO}^{3}$ and Zhice $\mathrm{Xu}^{1,2^{*}}$
}

\begin{abstract}
Background: Hypertension is one of primary clinical presentations of pre-eclampsia. The occurrence and progress of hypertension are closely related to vascular dysfunction. However, information is limited regarding the pathological changes of vascular functions in pre-eclamptic fetuses. Human umbilical cord vein was used to investigate the influence of pre-eclampsia on fetal blood vessels in this study.

Results: The present study found that the vasoconstriction responses to arginine vasopressin (AVP) and oxytocin (OXT) were attenuated in the pre-eclamptic umbilical vein as compared to in normal pregnancy, which was related to the downregulated AVP receptor 1a (AVPR1a), OXT receptor (OXTR), and protein kinase $C$ isoform $\beta$ (PKC $\beta$ ), owing to the deactivated gene transcription, respectively. The deactivated AVPR1a, OXTR, and PKCB gene transcription were respectively linked with an increased DNA methylation within the gene promoter.

Conclusions: To the best of our knowledge, this study first revealed that a hyper-methylation in gene promoter, leading to relatively reduced patterns of AVPR1a, OXTR, and PKCB expressions, which was responsible for the decreased sensitivity to AVP and OXT in the umbilical vein under conditions of pre-eclampsia. The data offered new and important information for further understanding the pathological features caused by pre-eclampsia in the fetal vascular system, as well as roles of epigenetic-mediated gene expression in umbilical vascular dysfunction.
\end{abstract}

Keywords: Pre-eclampsia, Arginine vasopressin, Oxytocin, DNA methylation, Umbilical vein dysfunction

\section{Background}

Pre-eclampsia (PE) is a leading cause of maternal morbidity, mortality, and premature birth in both developed and developing countries $[1,2]$. Although PE in women is a multi-systemic syndrome with unknown etiology, hypertension is a primary clinical presentation of PE. As a surrogate end point for vascular risk, vascular dysfunction is closely related to the occurrence and progress of hypertension. However, information regarding the pathological changes of vascular functions in pre-eclamptic fetuses is limited. The umbilical cord is a conduit between the developing fetus and placenta. Umbilical cord vessels are primary vascular structures that may reflect problems

\footnotetext{
* Correspondence: jennyqgao@126.com; xuzhice@suda.edu.cn

${ }^{\dagger}$ Qinqin Gao, Xiaorong Fan, and Ting Xu contributed equally to this work.

'Institute for Fetology and Department of Obstetrics and Gynecology, First

Hospital of Soochow University, Suzhou 215006, China

Full list of author information is available at the end of the article
}

originated from inadequate changes in maternal and fetal vascular systems. Human umbilical cord normally contains two arteries and one vein. The umbilical vein supplies the fetus with oxygenated, nutrient-rich blood from the placenta. Vascular functions of the umbilical vein are so important for placental-fetal circulation and fetal development in utero. Therefore, the present study was conducted with umbilical veins from healthy and preeclamptic pregnancy to investigate whether and how vascular functions would be affected under conditions of PE.

Because umbilical vessels have no autonomic innervation $[3,4]$, circulating and locally synthesized vasoactive substances are important in controlling vascular functions and blood flow in the placental-fetal circulation. As stress hormones, arginine vasopressin (AVP) and oxytocin (OXT) are mainly synthesized in the magnocellular neurons of the paraventricular and supraoptic nucleus of the

(c) The Author(s). 2019 Open Access This article is distributed under the terms of the Creative Commons Attribution 4.0 International License (http://creativecommons.org/licenses/by/4.0/), which permits unrestricted use, distribution, and 
hypothalamus. In most vascular beds, AVP and OXT are potent vasoconstrictors [5]. AVP has long been implicated in controlling blood pressure and vascular tone through binding of smooth muscle receptors (mainly classified into V1a (AVPR1a), V1b (AVPR1b), and V2 (AVPR2) subtypes) [6-8]. In normal delivery, high AVP concentrations in human umbilical cord blood have been reported $[9,10]$. Similarly, oxytocin (OXT), a nine amino acid neuropeptide, is also increased at late pregnancy and onset of labor [11, 12]. The actions of both central and peripheral OXT are mediated through oxytocin receptor (OXTR) [13]. It has been reported that AVP- and OXT-induced vasocontractions are mainly regulated by protein kinase $\mathrm{C}$ (PKC) pathway $[6,14$, 15].

In humans, high AVP and OXT concentrations are demonstrated in umbilical cord blood during normal delivery $[9,10,12]$. Do the high AVP and OXT in the circulation cause remarkable vasoconstrictions in umbilical vessels? Would AVP and OXT play the same physiological roles in pre-eclamptic umbilical vessels as they do in the normal ones? In fact, such is the paucity of knowledge of vascular reactivities of the umbilical vein, with very limited studies and information on umbilical vascular functions and none has compared umbilical vascular responses of AVP and OXT between PE and normal pregnancy. The present study, therefore, investigated the contractile responses of AVP and OXT in normal and pre-eclamptic umbilical vein, to reveal special features of umbilical vascular regulations and possible pathophysiological changes, as well as its underlying mechanisms under PE condition. The data gained in the present study provided new and critical information on regulations of umbilical vascular functions under preeclamptic conditions that in favor of further understanding the pathological features and mechanisms of PE as well as vascular diseases in fetal origins.

\section{Results}

AVP or OXT-induced contractions in human umbilical vein Both AVP and OXT could induce dose-dependent constrictions in human umbilical vein (HUV) (Fig. 1a, d). There were no significant differences in $\mathrm{KCl}$-induced maximal contraction between NP and PE group (Fig. 1b, e), whereas, the Emax (AVP- or OXT-induced contraction at $\left.10^{-4} \mathrm{~mol} / \mathrm{L}\right)$ and $\mathrm{pD} 2(-\log [50 \%$ effective concentration]) values for AVP and OXT were significantly decreased in pre-eclamptic umbilical vein (Fig. 1c, f). These data indicated that pre-eclamptic umbilical vein was significantly insensitive to AVP and OXT.

\section{Expression of AVP or OXT receptors in human umbilical vein}

In the vasculature, AVP receptors include AVPR1a, AVPR1b, and AVPR2 [7]. Compared with NP, mRNA and protein levels of AVPR1a, not AVPR2, were decreased in the PE group (Fig. 2a, b). SR49059 (AVPR1aspecific antagonist) completely blocked AVP-mediated contractions in both NP and PE groups and without significant differences in AVP-induced vasoconstrictions between the two groups after pretreatment with SR49059 (Fig. 2c). Similarly, as shown in Fig. 2d and e, there was a significant decrease in mRNA and protein of OXTR in the PE group. Meanwhile, OXTR-specific antagonist (atosiban) could completely block OXTmediated contractions in the umbilical vein, without significant differences between NP and PE groups after pretreated with atosiban (Fig. 2f). These data indicated that the decreased sensitivity of pre-eclamptic umbilical vein to AVP and OXT was related to the downregulated AVPR1a and OXTR due to the deactivated gene transcription, respectively.

\section{The decreased sensitivity of AVP and OXT was also dependent on PKC pathway}

AVP- and OXT-induced vasocontractions are mainly regulated by PKC pathway $[6,14,15]$. As shown in Fig. 3a, PKC agonist (PDBu) caused weaker dosedependent contractions in pre-eclamptic HUV than that of NP group. In the vasculature, PKC mainly includes $\alpha$, $\beta, \gamma, \delta$, and $\varepsilon$ isoforms [16]. There were no significant differences in $\mathrm{PKC} \alpha, \mathrm{PKC} \gamma, \mathrm{PKC} \varepsilon$, and PKC $\delta$ mRNA expression between NP and PE group; however, mRNA levels of $P K C \beta$ were significantly decreased in PE compared with that in NP group (Fig. 3b). Protein levels of PKC $\beta$ were also significantly decreased in pre-eclamptic HUV (Fig. 3c). Meanwhile, PKC-specific antagonist (GF109203X) could restrain AVP- or OXT-induced vasoconstrictions in both NP and PE groups, without significant differences in AVP- or OXT-mediated vasoconstrictions between $\mathrm{NP}$ and $\mathrm{PE}$ group following pretreatment with GF109203X (Fig. 3d, e). Meanwhile, GF109203X could produce a weaker inhibitory effect on AVP- or OXT-mediated vasoconstrictions in NP group (Fig. 3d, e). These data indicated that the decreased sensitivity of pre-eclamptic umbilical vein to AVP and OXT was also related to the downregulated PKC pathway.

\section{DNA methylation of $\mathrm{CpG}$ locus within AVPR1a gene promoter in human umbilical vein}

AVPR1a is located on chromosome 12q14.2. To clarify whether the deactivated transcription of AVPR1a was associated with DNA methylation alterations, we assessed changes of AVPR1a transcription after adding 5-Aza-2' deoxycytidine (5-Aza, a specific DNA methylation transferase inhibitor) in human umbilical cord vein endothelial cells (HUVECs). In HUVECs, 5-Aza treatment significantly increased AVPR1a gene transcription (Fig. 4b). One CpG island contains $14 \mathrm{CpG}$ sites within 


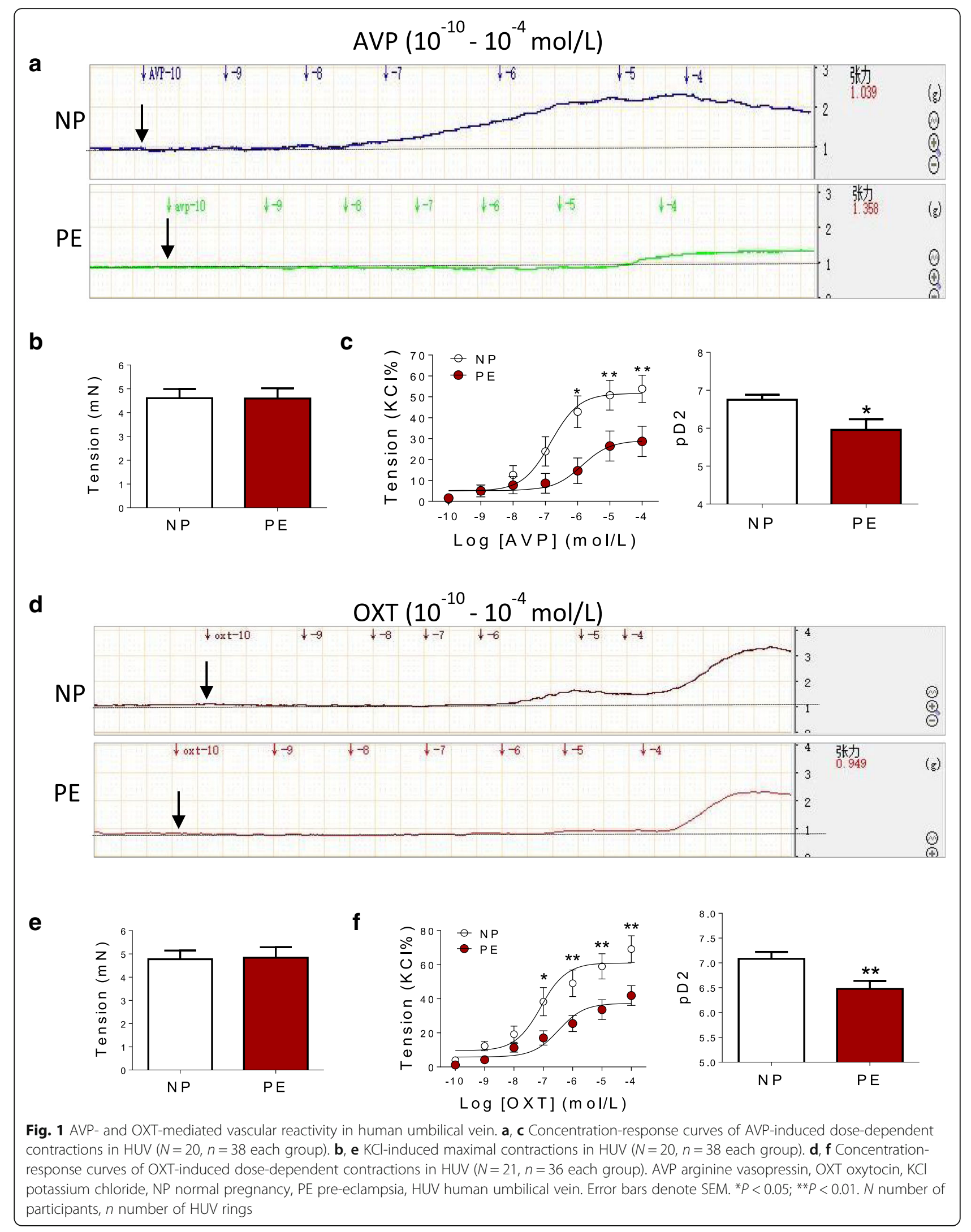




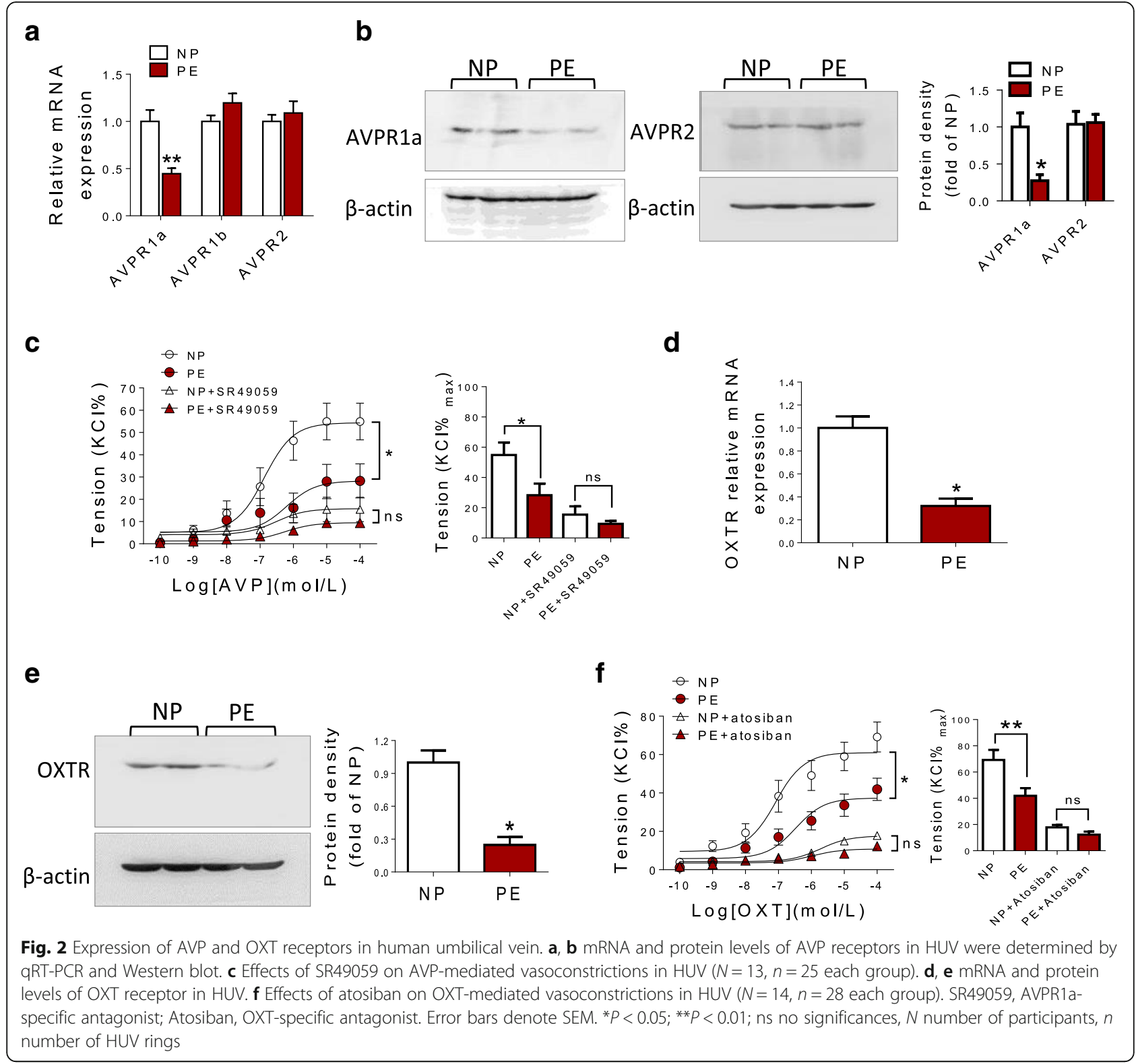

exon of AVPR1a gene (Fig. 4a). Table 1 showed CpG labels. Next, we validated methylation levels of these 14 CpG sites by targeted bisulfite sequencing. The bisulfite conversion rate of each sample was higher than $99 \%$, and no significant difference was observed between NP and PE group, indicating bisulfite conversion was efficient and reliable in the experiments (Fig. 4c). Compared with NP, the mean methylation percentage of these 14 CpG sites in pre-eclamptic umbilical vein was significantly increased with specific CpG site 5 and 6 (Fig. 4de, Table 2). Correlation analysis between AVPR1a gene methylation and expression was also conducted. There was a significantly inverse correlation between the methylation statuses of CpG sites (5 and 6) in AVPR1a gene promoter and AVPR1a gene expression (Fig. 4f). In normal and pre-eclamptic HUVECs, after 5-Aza treatment, mRNA levels of AVPR1a were significantly increased and without significant differences between the two groups (Fig. 4g).

\section{DNA methylation of CpG locus within OXTR gene} promoter in human umbilical vein

OXTR is located on chromosome 3p25. Sequence analysis identified one CpG island that contains $22 \mathrm{CpG}$ sites within exons of the OXTR gene (Fig. 5a). Table 3 provided a key for CpG labels. In HUVECs, after 5-Aza treatment, OXTR mRNA level was significantly increased (Fig. 5b). Compared with NP, mean methylation percentage of the total $22 \mathrm{CpG}$ sites in the PE group was remarkably increased (Fig. 5d), whereas, no significant 


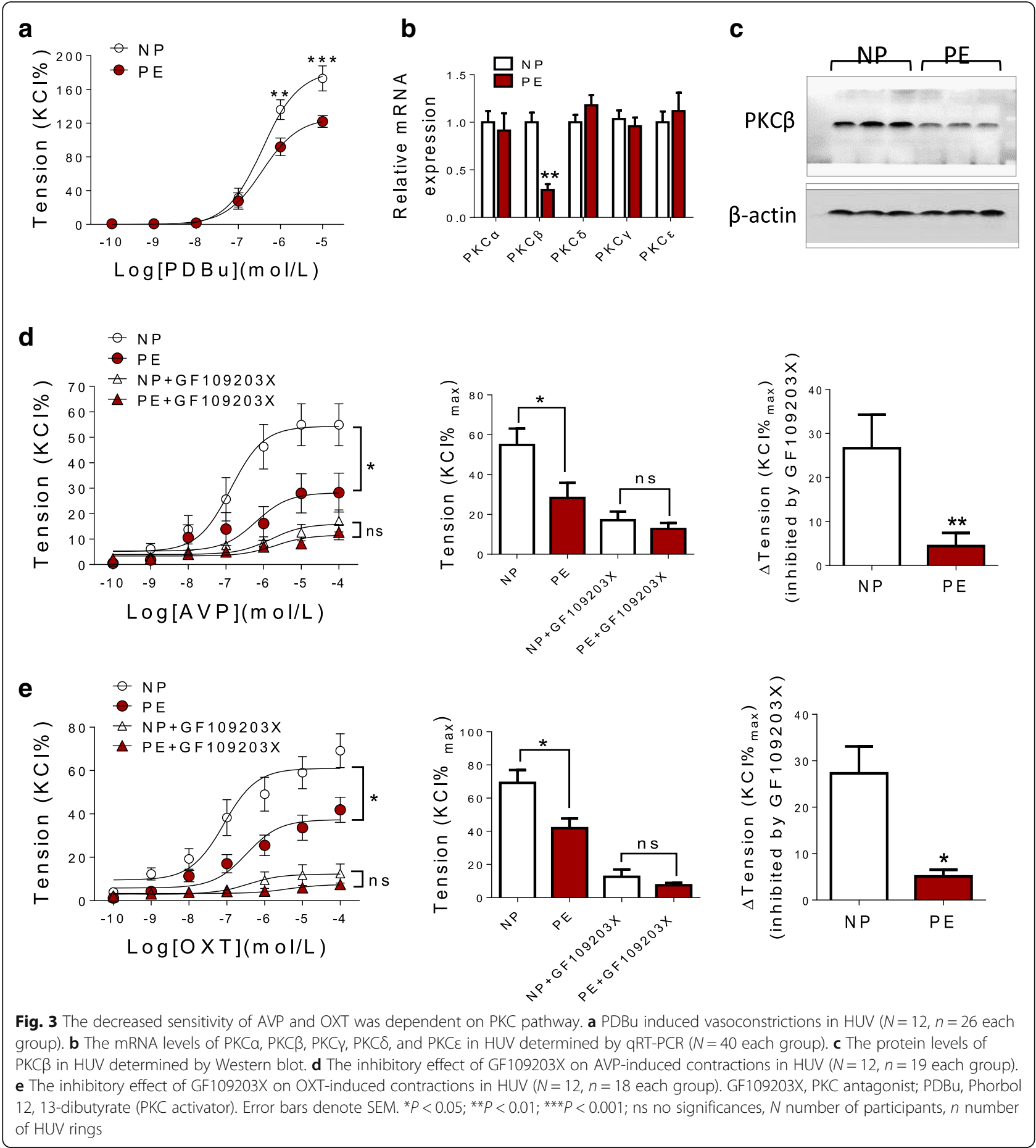

differences were observed in each tested CpG site between NP and PE group (Fig. 5c, d). Table 4 showed the position and methylation levels of these $22 \mathrm{CpG}$ sites. As shown in Fig. 5e, there was a significantly inverse correlation between the methylation status of $22 \mathrm{CpG}$ sites in OXTR gene promoter and OXTR gene expression. In normal and pre-eclamptic HUVECs, mRNA levels of OXTR were significantly increased, and no significant differences were observed between the two groups after 5-Aza treatment (Fig. 5g).

\section{DNA methylation of CpG locus within PKCB gene promoter in human umbilical vein}

$P K C B$ is located on chromosome 16p12.2. One CpG island contains 44 CpG sites within exons of $P K C B$ gene (Fig. 6a). 5-Aza treatment also significantly increased 


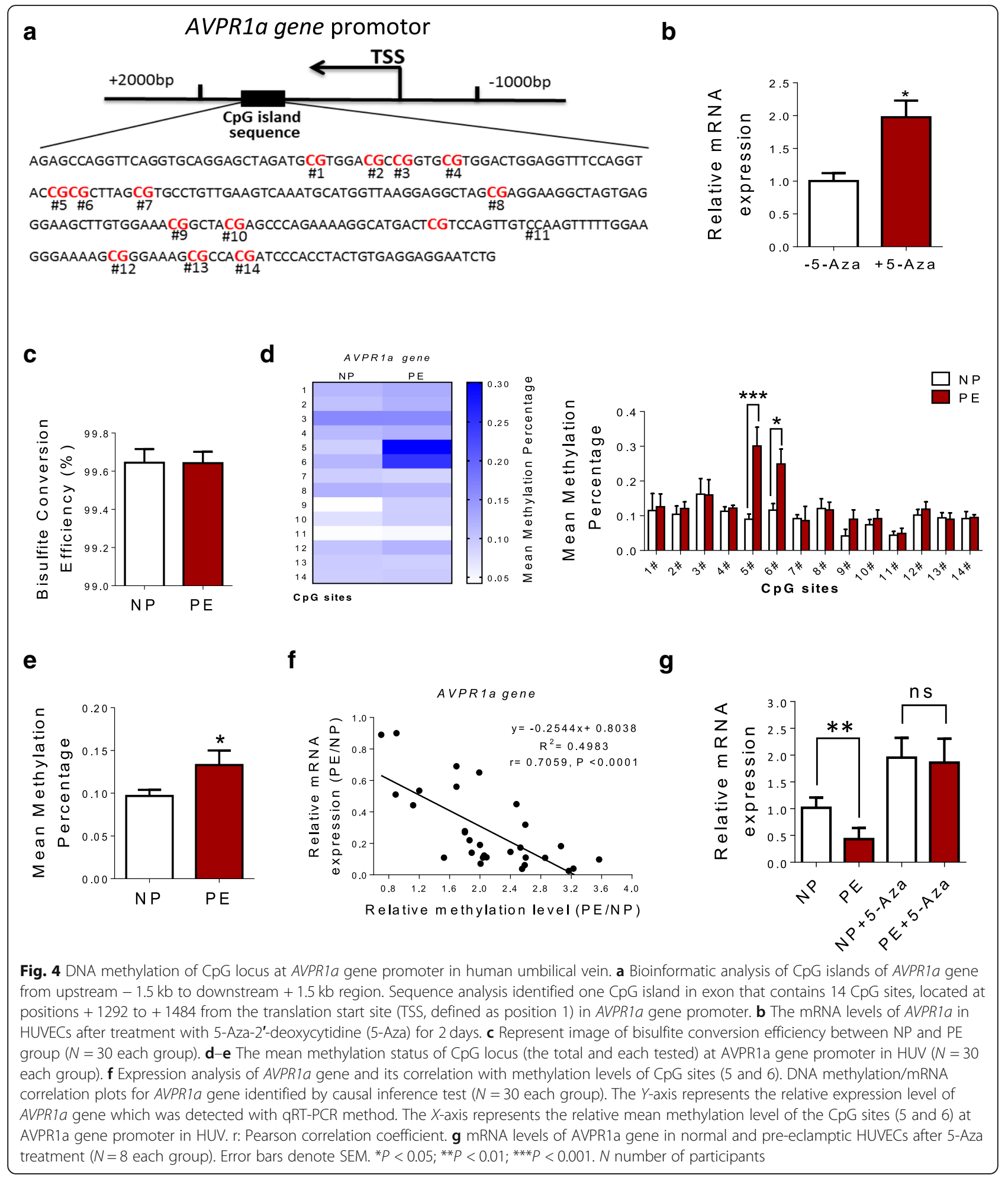

PKCB gene transcription in HUVECs (Fig. 6b). Targeted bisulfite sequencing showed that compared with NP, the mean methylation percentage of the total $44 \mathrm{CpG}$ sites was remarkably increased with specific CpG sites (3841) within $P K C B$ gene promoter in the $\mathrm{PE}$ group, whereas no significant difference was observed in other specific CpG sites between NP and PE group (Fig. 6c-e). Position and methylation levels of the 44 CpG sites were listed in Table 5. After careful analysis of DNA methylation and expression data, it is concluded that there was 
Table 1 Methylated CpG sites at AVPR1a gene promoter measured in this study

\begin{tabular}{llll}
\hline Gene & Position & Genomic location & Relative to TSS, bp \\
\hline AVPR1a & 1 & Chr12: 63545106 & +1484 \\
2 & Chr12: 63545111 & +1479 \\
3 & Chr12: 63545119 & +1471 \\
4 & Chr12: 63545152 & +1438 \\
5 & Chr12: 63545174 & +1416 \\
6 & Chr12: 63545180 & +1410 \\
7 & Chr12: 63545212 & +1378 \\
8 & Chr12: 63545251 & +1339 \\
9 & Chr12: 63545258 & +1332 \\
10 & Chr12: 63545260 & +1330 \\
11 & Chr12: 63545284 & +1306 \\
12 & Chr12: 63545289 & +1301 \\
13 & Chr12: 63545292 & +1298 \\
14 & Chr12: 63545298 & +1292 \\
\hline
\end{tabular}

also a significantly inverse correlation between the methylation statuses of 38-41 CpG sites and PKCB expression (Fig. 6f). After 5-Aza treatment, mRNA levels of $P K C B$ were significantly increased, and no significant differences were observed between normal and preeclamptic HUVECs (Fig. 6g).

\section{Discussion}

This present study found a special feature of AVP- and OXT-mediated vascular contractions in pre-eclamptic umbilical vasculature. The main findings are as follows:

Table 2 The methylation status of CpG locus (the total and each tested) at AVPR1a gene promoter. The data was expressed as mean \pm SEM. PE pre-eclampsia, NP normal pregnant. ${ }^{* *} P<$ $0.01 ;{ }^{* * *} P<0.001$

\begin{tabular}{llll}
\hline Gene & Position & NP & PE \\
\hline AVPR1a & 1 & $0.104 \pm 0.028$ & $0.123 \pm 0.028$ \\
2 & $0.107 \pm 0.032$ & $0.119 \pm 0.021$ \\
3 & $0.165 \pm 0.027$ & $0.163 \pm 0.010$ \\
4 & $0.115 \pm 0.015$ & $0.122 \pm 0.009$ \\
5 & $0.094 \pm 0.015$ & $0.291 \pm 0.064^{* *}$ \\
6 & $0.112 \pm 0.019$ & $0.280 \pm 0.066^{* * *}$ \\
7 & $0.092 \pm 0.039$ & $0.083 \pm 0.014$ \\
8 & $0.131 \pm 0.028$ & $0.111 \pm 0.031$ \\
9 & $0.047 \pm 0.019$ & $0.088 \pm 0.026$ \\
10 & $0.072 \pm 0.018$ & $0.088 \pm 0.028$ \\
& 11 & $0.043 \pm 0.011$ & $0.049 \pm 0.016$ \\
12 & $0.096 \pm 0.019$ & $0.131 \pm 0.015$ \\
& 13 & $0.102 \pm 0.040$ & $0.100 \pm 0.034$ \\
& 14 & $0.094 \pm 0.021$ & $0.100 \pm 0.004$ \\
Average & $0.098 \pm 0.013$ & $0.132 \pm 0.017^{*}$ \\
\hline
\end{tabular}

(1) Compared with the normal control, the vasoconstriction responses to AVP and OXT were attenuated in preeclamptic umbilical vein, which was related to the downregulated AVPR1a, OXTR, and PKCB, owing to the deactivated gene transcription, respectively. (2) The deactivated AVPR1a, OXTR, and PKCB transcriptions were respectively linked with an increased DNA methylation within gene promoter. The data gained not only offered new information for further understanding the pathological features and mechanisms of pre-eclamptic umbilical cords, but also providing novel clues for roles of epigenetic-mediated gene expression in fetal vascular dysfunction.

Although it is well known that AVP and OXT can produce vascular contractions in adults $[5,17]$, data regarding their functional effects on fetal blood vessels is limited. In human, umbilical vessels are only healthy fetal blood vessels that can be obtained ethically in medical studies. Both of AVP and OXT exhibited significant dose-dependent vasoconstrictions in human fetal umbilical vein, suggesting that the two peptides are critically involved in the regulating umbilical vascular tone and circulation. Notably, we found that compared with normal pregnancy, pre-eclamptic umbilical vein was significantly insensitive to AVP and OXT, which was not only associated with their respective receptors, but also correlated with PKC pathway. This finding was supported by the following data: (1) The mRNA and protein levels of AVPR1a and OXTR were remarkably decreased in preeclamptic umbilical vein. Meanwhile, AVPR1a- or OXTR-specific antagonist could completely block AVPand OXT-mediated contractions in umbilical vein, respectively. (2) PKC agonist caused weaker dosedependent contractions, and $\mathrm{PKC}$ antagonist produced a weaker inhibitory effect on AVP- and OXT-mediated vasoconstrictions in pre-eclamptic umbilical vein; furthermore, mRNA and protein levels of PKC $\beta$ were significantly decreased in pre-eclamptic umbilical vein. These data above indicated that the decreased sensitivity of pre-eclamptic umbilical vein to AVP and OXT was related to the downregulated AVPR1a, OXTR, and PKCB, particularly with their deactivated gene transcription.

The number of studies in humans and laboratory animals indicated promoter DNA methylation levels are important for transcriptional regulation of AVPR1a [1820], OXTR [21-23], and PKCB [24-27]. In exploring the possible underlying mechanisms of the altered AVP- and OXT-mediated vascular functions, the present study also focused on epigenetic causes. Firstly, to clarify whether the deactivated transcriptions of $A V P R 1 a, O X T R$, and $P K C B$ are owing to DNA methylation, we assessed the changes of these gene transcriptions after adding 5-Aza in HUVECs. 5-Aza treatment significantly increased $A V P R 1 a, O X T R$, and $P K C B$ gene transcriptions, 


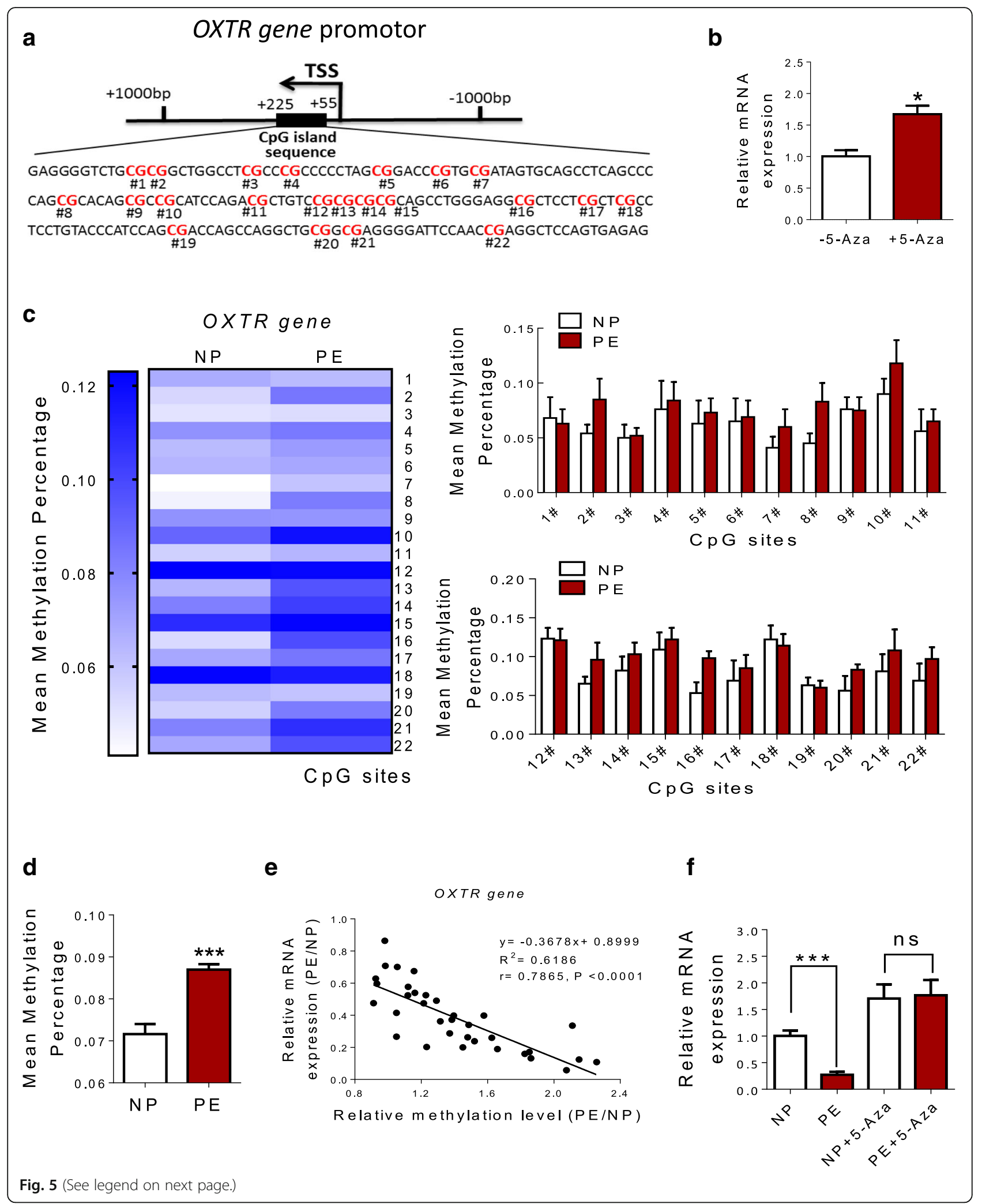

indicating that these gene transcriptions were regulated by DNA methylation in human umbilical vascular cells. Secondly, we evaluated DNA methylation status of $\mathrm{CpG}$ sites within AVPR1a, OXTR, and PKCB gene promoter and found that the mean methylation percentages of CpG sites within CpG islands in AVPR1a, OXTR, and 
(See figure on previous page.)

Fig. 5 DNA methylation of CpG locus at OXTR gene promoter in human umbilical vein. a Sequence analysis identified one CpG island in exon 1 that contains $22 \mathrm{CpG}$ sites, located at positions +55 to +225 from the TSS in the OXTR gene promoter. $\mathbf{b}$ mRNA levels of OXTR in HUVECs after treatment with 5-Aza. c-d Represent the mean methylation status of the genomic regions in OXTR gene promoter. Each bar represents mean methylation percentage in a genomic region of a sample. e Expression analysis of OXTR gene and its correlation with methylation levels of 22 CpG sites. DNA methylation/mRNA correlation plots for OXTR gene identified by causal inference test. The $y$-axis represents the relative expression level of OXTR gene which was detected with qRT-PCR method. The $x$-axis represents the relative mean methylation level of all the 22 CpG sites in OXTR gene. r: Pearson correlation coefficient. $\mathbf{f}$ mRNA levels of OXTR gene in normal and pre-eclamptic HUVECs after 5-Aza treatment $\left(N=8\right.$ each group). Error bars denote SEM. ${ }^{*} P<0.05 ;{ }^{* *} P<0.001 . N$ number of participants

$P K C B$ gene promoter were obviously increased in umbilical vein under conditions of PE. Thirdly, we conducted correlation analysis between gene methylation and expression and found that there was a significantly inverse correlation between DNA methylation levels of gene promoter and gene transcription. Fourthly, we isolated and cultured HUVECs in vitro and evaluated expressions of these genes in both of normal and preeclamptic HUVECs after 5-Aza treatment. Compared with normal, mRNA levels of these genes were decreased in the pre-eclamptic HUVECs. After 5-Aza treatment, mRNA levels of these genes were significantly increased in both of normal and pre-eclamptic HUVECs, and no significant differences were observed in mRNA

Table 3 Methylated CpG sites at OXTR gene promoter measured in this study

\begin{tabular}{|c|c|c|c|}
\hline Gene & Position & Genomic location & Relative to TSS, bp \\
\hline \multirow[t]{22}{*}{ OXTR } & 1 & Chr3: 8811075 & +225 \\
\hline & 2 & Chr3: 8811090 & +210 \\
\hline & 3 & Chr3: 8811093 & +207 \\
\hline & 4 & Chr3: 8811108 & +192 \\
\hline & 5 & Chr3: 8811128 & +172 \\
\hline & 6 & Chr3: 8811132 & +168 \\
\hline & 7 & Chr3: 8811139 & +161 \\
\hline & 8 & Chr3: 8811153 & +147 \\
\hline & 9 & Chr3: 8811155 & +145 \\
\hline & 10 & Chr3: 8811157 & +143 \\
\hline & 11 & Chr3: 8811159 & +141 \\
\hline & 12 & Chr3: 8811166 & +134 \\
\hline & 13 & Chr3: 8811176 & +124 \\
\hline & 14 & Chr3: 8811179 & +121 \\
\hline & 15 & Chr3: 8811186 & +114 \\
\hline & 16 & Chr3: 8811209 & +91 \\
\hline & 17 & Chr3: 8811213 & +87 \\
\hline & 18 & Chr3: 8811219 & +81 \\
\hline & 19 & Chr3: 8811229 & +71 \\
\hline & 20 & Chr3: 8811233 & +67 \\
\hline & 21 & Chr3: 8811243 & +57 \\
\hline & 22 & Chr3: 8811245 & +55 \\
\hline
\end{tabular}

levels of these genes between the two groups. Together, the present study first indicated that transcriptions of AVPR1a, OXTR, and PKCB were regulated by DNA methylation in human umbilical vessels and revealed that hyper-methylation in AVPR1a, OXTR, and PKCB gene promoter, leading to a relatively low pattern of gene expressions, were responsible for the decreased sensitivity of AVP and OXT in pre-eclamptic umbilical vessels. Large amount of research showed that DNA methylation has been considered for contribution to the development for PE [28]. In the present study, we first demonstrated that DNA methylation-mediated gene

Table 4 The methylation status of CpG locus (the total and each tested) at OXTR gene promoter. The data was expressed as mean \pm SEM. PE pre-eclampsia, NP normal pregnant. ***P<0.001

\begin{tabular}{|c|c|c|c|}
\hline Gene & Position & NP & $P E$ \\
\hline \multirow[t]{23}{*}{ OXTR } & 1 & $0.068 \pm 0.019$ & $0.063 \pm 0.013$ \\
\hline & 2 & $0.054 \pm 0.008$ & $0.085 \pm 0.019$ \\
\hline & 3 & $0.050 \pm 0.012$ & $0.052 \pm 0.007$ \\
\hline & 4 & $0.076 \pm 0.026$ & $0.084 \pm 0.017$ \\
\hline & 5 & $0.063 \pm 0.021$ & $0.073 \pm 0.013$ \\
\hline & 6 & $0.065 \pm 0.021$ & $0.069 \pm 0.015$ \\
\hline & 7 & $0.041 \pm 0.010$ & $0.060 \pm 0.016$ \\
\hline & 8 & $0.045 \pm 0.004$ & $0.083 \pm 0.017$ \\
\hline & 9 & $0.076 \pm 0.011$ & $0.075 \pm 0.012$ \\
\hline & 10 & $0.090 \pm 0.014$ & $0.118 \pm 0.021$ \\
\hline & 11 & $0.056 \pm 0.020$ & $0.065 \pm 0.011$ \\
\hline & 12 & $0.123 \pm 0.014$ & $0.121 \pm 0.015$ \\
\hline & 13 & $0.065 \pm 0.009$ & $0.096 \pm 0.022$ \\
\hline & 14 & $0.082 \pm 0.018$ & $0.103 \pm 0.015$ \\
\hline & 15 & $0.109 \pm 0.022$ & $0.122 \pm 0.015$ \\
\hline & 16 & $0.053 \pm 0.014$ & $0.098 \pm 0.009$ \\
\hline & 17 & $0.069 \pm 0.026$ & $0.085 \pm 0.017$ \\
\hline & 18 & $0.122 \pm 0.018$ & $0.114 \pm 0.015$ \\
\hline & 19 & $0.063 \pm 0.010$ & $0.060 \pm 0.009$ \\
\hline & 20 & $0.056 \pm 0.019$ & $0.083 \pm 0.007$ \\
\hline & 21 & $0.081 \pm 0.022$ & $0.108 \pm 0.027$ \\
\hline & 22 & $0.069 \pm 0.022$ & $0.097 \pm 0.015$ \\
\hline & Average & $0.0716 \pm 0.0024$ & $0.0870 \pm 0.0013^{* * *}$ \\
\hline
\end{tabular}




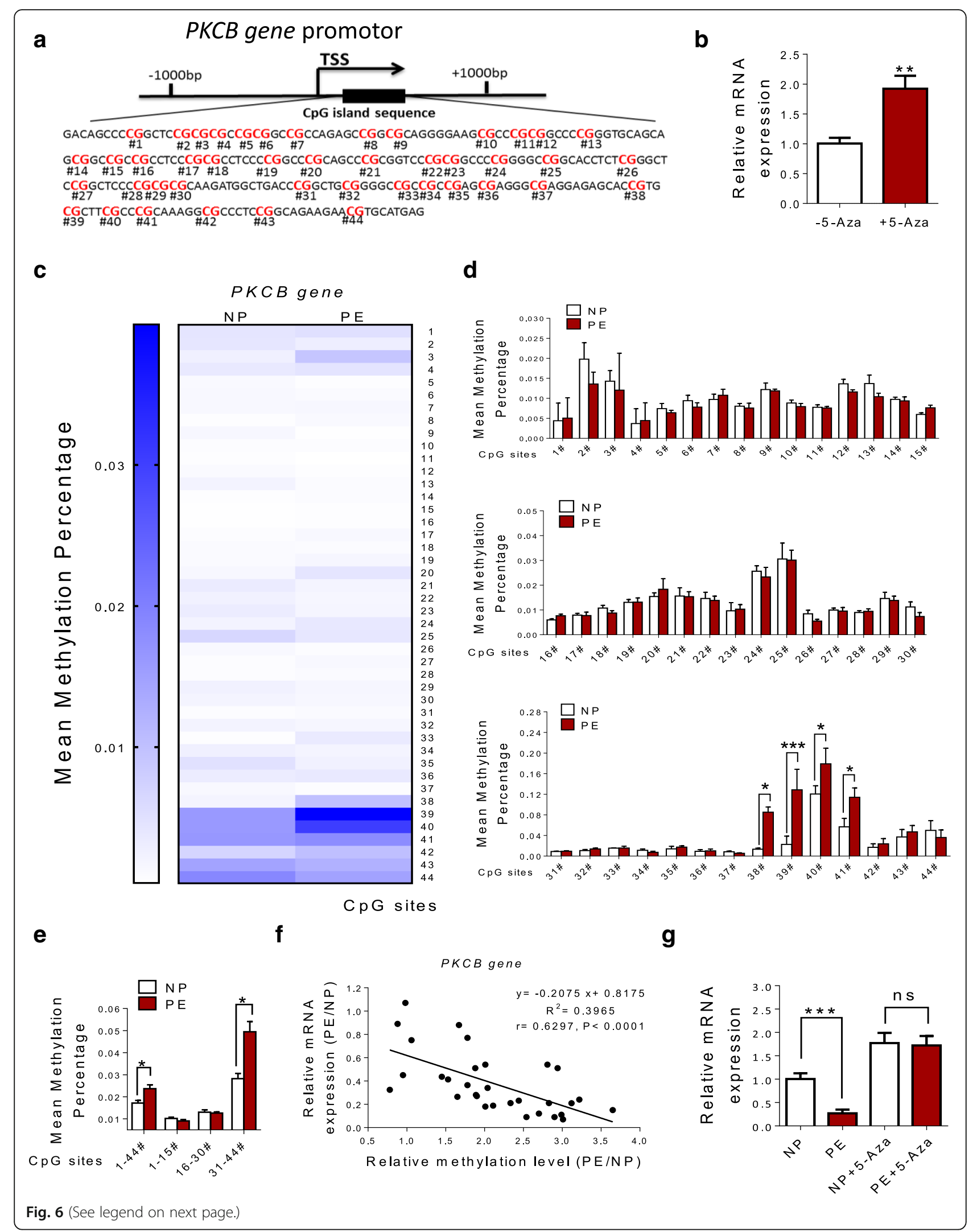


(See figure on previous page.)

Fig. 6 DNA methylation of CpG locus at PKCB gene promoter in human umbilical vein. a One CpG island that contains 44 CpG sites, located at positions +27 to +321 from TSS at PKCB gene promoter. $\mathbf{b}$ mRNA levels of PKCB in HUVECs after treatment with 5-Aza. c-e The mean methylation status of $C p G$ locus (the total and each tested) at $P K C B$ gene promoter in HUV ( $N=30$ each group). $\mathbf{f}$ DNA methylation/mRNA correlation plots for PKCB gene identified by causal inference test $(N=30$ each group). $\mathbf{g}$ mRNA levels of OXTR gene in normal and pre-eclamptic HUVECs after 5-Aza treatment ( $N=8$ each group). Error bars denote SEM. ${ }^{*} P<0.05 ;{ }^{* *} P<0.01$; ${ }^{* *} P<0.001$. N number of participants

expression was also critically involved in the pathogenesis of vascular dysfunction in pre-eclamptic umbilical vasculature. The pathological and clinical importance of DNA methylation in pre-eclamptic vascular dysfunction deserves further investigation.

Significance of our findings is also closely linked to "the development of chronic diseases in fetal origins." According to this theory, prenatal adverse factors have been demonstrated as major causes to induce diseases after birth [29-31]. PE could act as a stress for development fetuses. Thirty percent of newborns born from pre-eclamptic women experience some forms of adverse prenatal outcome, including prematurity and intrauterine growth retardation $[1,2]$. PE is a long-term disease risk factor for the mother and possibly the offspring too [32-34]. Evidence from clinical studies has proposed that children born from pre-eclamptic women have a higher risk of suffering neurological, psychological, and behavioral alterations, particularly cardiovascular diseases, including hypertension and stroke, compared to children born from normal pregnancies [32, 33, 35-40]. However, to date, the mechanisms behind these vascular outcomes are poorly understood. In human, the

Table 5 The methylation status of CpG locus (the total and each tested) at $P K C B$ gene promoter. The data was expressed as mean \pm SEM. PE pre-eclampsia, NP normal pregnant. ${ }^{*} P<0.05$; ${ }^{* *} P<0.01$; ${ }^{* *} P<0.001$

\begin{tabular}{llll}
\hline Gene & Position & NP & PE \\
\hline PKCB & 31 & $0.009 \pm 0.0003$ & $0.007 \pm 0.0008$ \\
& 32 & $0.009 \pm 0.0013$ & $0.011 \pm 0.0013$ \\
33 & $0.010 \pm 0.0008$ & $0.009 \pm 0.0007$ \\
34 & $0.014 \pm 0.0003$ & $0.013 \pm 0.0007$ \\
35 & $0.008 \pm 0.0006$ & $0.011 \pm 0.0018$ \\
36 & $0.007 \pm 0.0008$ & $0.008 \pm 0.0009$ \\
37 & $0.008 \pm 0.0006$ & $0.009 \pm 0.0007$ \\
38 & $0.016 \pm 0.0048$ & $0.084 \pm 0.0156^{*}$ \\
39 & $0.021 \pm 0.0095$ & $0.108 \pm 0.0166^{* * *}$ \\
& 40 & $0.121 \pm 0.0696$ & $0.186 \pm 0.0536^{*}$ \\
& 41 & $0.063 \pm 0.0234$ & $0.134 \pm 0.0237^{* *}$ \\
42 & $0.014 \pm 0.0018$ & $0.014 \pm 0.0016$ \\
& 43 & $0.028 \pm 0.0117$ & $0.043 \pm 0.0178$ \\
& 44 & $0.008 \pm 0.0011$ & $0.008 \pm 0.0010$ \\
Average & $0.024 \pm 0.0038$ & $0.046 \pm 0.0096^{* *}$ \\
\hline
\end{tabular}

umbilical cord is physiologically and genetically part of the fetus and may reflect problems originated from inadequate changes in the fetus with maternal history of PE. Interestingly, this study found that pre-eclamptic fetal umbilical vein showed a specific epigenetic-mediated vascular dysfunction, suggesting that pre-eclamptic fetal vascular system may undergo similar changes as it is represented in fetal umbilical vessels. It is rational that there may exist the same or similar abnormalities in preeclamptic fetal vascular systems as that observed in the fetal umbilical cord vein. Given this, due to epigenetic code that can be inherited, it put forward the hypothesis that the child with maternal history of PE are with a higher risk of diseases and disorders particularly in vascular problems. Recent cohort studies assessing whether maternal PE are associated with vascular problems in the offspring throughout childhood and early adolescence have provided supportive evidence for this concept [39-42]. Although this study did not investigate the offspring, the interesting finding in epigenetic-mediated umbilical vascular dysfunctions provides new important information for further studies on cardiovascular diseases in fetal origins.

In conclusion, this study firstly revealed a special feature of vascular regulations and pathophysiological changes in the umbilical vein under conditions of PE. Significances of the finding includes (1) offering new information for further understanding the pathological features of pre-eclamptic fetal umbilical vessels, and (2) underlining roles of epigenetic-mediated gene expression in pre-eclamptic umbilical vascular dysfunction, and (3) providing new insights into the underlying mechanisms of PE-related higher risks of vascular diseases and disorders in fetal origins. In addition, it is well known that an altered placenta-umbilical cord circulation plays an important role in the development of PE $[2,43]$. Whether and how the changed umbilical vascular dysfunction contributes or complicates to PE is another interesting and important direction for researching.

\section{Materials and methods}

\section{Sample preparation}

Healthy normal pregnant $(N=42)$ and pre-eclamptic women $(N=40)$ were recruited from the local hospitals, Suzhou, China. The Ethics Committee of the First Hospital of Soochow University approved all procedures in this work (ref. no. 2015-129), and all participants were 
given informed consent. Healthy pregnant participants were defined as blood pressure $<120 / 90 \mathrm{mmHg}$ and no clinically significant complications. Pre-eclamptic pregnant participants were defined as blood pressure $>140$ / $90 \mathrm{mmHg}$ and significant proteinuria after the 20th weeks of pregnancy [1, 44]. Women with essential hypertension or medical complications, such as diabetes and renal and cardiovascular diseases, were excluded from the study. The clinical characteristics of all participants were detailed in Table 6.

\section{Measurement of vascular tension}

Human umbilical cords were immediately acquired from normal and pre-eclamptic pregnant women after vaginal delivery and delivered within $1 \mathrm{~h}$. The umbilical cords $(10 \mathrm{~cm}$ in length) were kept in iced Krebs solution (containing in mmol/L $\mathrm{NaCl} 119, \mathrm{NaHCO}_{3} 25, \mathrm{KH}_{2} \mathrm{PO}_{4}$ 1.2, $\mathrm{KCl} 4.7, \mathrm{MgSO}_{4}$ 1.0, glucose 11, and $\mathrm{CaCl}_{2} 2.5$ ), and bubbled with $95 \% \mathrm{O}_{2}$ and $5 \% \mathrm{CO}_{2}$. Human umbilical vein was carefully isolated and cut into rings approximately $4-5 \mathrm{~mm}$ in length and suspended in a $5 \mathrm{~mL}$ organ bath with $5 \mathrm{~mL}$ Krebs solution and continuously with a mixture of $95 \% \mathrm{O}_{2}$ and $5 \% \mathrm{CO}_{2}$. Under the tension of $2 \mathrm{~g}$, HUV rings were allowed to balance for $2 \mathrm{~h}$. Then the contraction of potassium chloride $(\mathrm{KCl}, 120$ $\mathrm{mol} / \mathrm{L}$ ) was used to gain maximum vascular reaction. The contraction induced by AVP or OXT was standardized through comparing with the maximal tension caused by $\mathrm{KCl}$. HUV rings were contracted by the addition of incremental doses of vasopressin AVP $\left(10^{-10}\right.$ to $\left.10^{-4} \mathrm{~mol} / \mathrm{L}\right)$, OXT $\left(10^{-10}\right.$ to $\left.10^{-4} \mathrm{~mol} / \mathrm{L}\right)$, or $\mathrm{PDBu}$ (Phorbol 12, 13-dibutyrate, PKC activator; $10^{-10}$ to $10^{-5}$ $\mathrm{mol} / \mathrm{L}$ ) at 4-min intervals. Between continuous concentrations of AVP, OXT, or PDBu, there was at least 4 min of reaction time, during that period, the reaction of preceding concentration reached equilibrium phase. In the subsequent experiment, SR49059 (a specific inhibitor of AVP, $10 \mu \mathrm{mol} / \mathrm{L}$ ), atosiban (a specific inhibitor of OXT,

Table 6 Basic characteristics of pre-eclampsia cases and normotensive controls

\begin{tabular}{lll}
\hline Characteristics & NP & PE \\
\hline Number of subjects & 42 & 40 \\
Maternal age (year) & $28.40 \pm 4.50$ & $28.20 \pm 4.10$ \\
Gestational age (week) & $38.4 \pm 2.1$ & $33.3 \pm 4.1^{* *}$ \\
Birth weight (kg) & $3.2 \pm 0.8$ & $2.6 \pm 0.8^{*}$ \\
Systolic BP (mmHg) & $107.6 \pm 7.9$ & $164.8 \pm 19.2^{* *}$ \\
Diastolic BP (mmHg) & $79.5 \pm 9.8$ & $105.4 \pm 12.1^{* *}$ \\
Proteinuria (g/24h) & $0.17 \pm 0.05$ & $5.01 \pm 2.62^{* *}$ \\
S/D ratio & $2.02 \pm 0.44$ & $3.82 \pm 1.86^{*}$ \\
\hline
\end{tabular}

The data was expressed as mean \pm SD. Pre-eclampsia vs. normal pregnant. S/D ratio, ratio of systolic and diastolic blood flow in the umbilical artery. PE preeclampsia, $N P$ normal pregnant. ${ }^{* *} P<0.01 ;{ }^{* *} P<0.001$
$10 \mu \mathrm{mol} / \mathrm{L}$ ), or GF109203X (PKC antagonist, $100 \mu \mathrm{mol} /$ L) were used for pretreating HUV rings for $30 \mathrm{~min}$ before application of AVP or OXT, and vessel responses were recorded [45]. All drugs were freshly prepared and purchased from Sigma-Aldrich (St. Louis, MO).

\section{Quantitative real-time PCR (qRT-PCR) and Western blot analysis}

Total RNA was isolated from HUV using Trizol reagent and was reversed transcribed using first-strand cDNA Synthesis Kit (Invitrogen). qRT-PCR was performed using SYBR Green Supermix Taq Kit (Takara Biotechnology Co., Ltd., Dalian, China) and analyzed on an iQ5 Real-Time PCR Detection System (Bio-Rad Laboratories, Inc., Hercules, CA, USA). The primer sequences are listed in Table 7. $\Delta \Delta \mathrm{Ct}$ method was used to comparatively quantify the amount of mRNA levels. The protein abundance of AVPR1a, AVPR2, OXTR, and PKC ( $\alpha$ and $\beta)$ in HUV was measured with Western blot normalized to $\beta$-actin. The primary antibodies were the rabbit polyclonal antibody (Santa Cruz Biotechnology) against AVPR1a, AVPR2, OXTR, or PKC $\beta$ (all 1:1000). The secondary antibody was the goat anti-rabbit antibody (1:500; Beyotime Biotechnology, Jiangsu, China). Immuno-signals were visualized using UVP imaging system (EC3-ImagingSystem, Upland, CA, USA). Imaging signals were calculated and analyzed, and then the ratio of band brightness to $\beta$-actin was acquired to measure relative protein expression levels as previously described $[46,47]$.

\section{DNA isolation and targeted bisulfite sequencing assay}

To prepare genomic DNA, HUV rings were lysed with lysis buffer containing (10 mM Tris-Cl (pH 7.5), $10 \mathrm{mM}$ $\mathrm{NaCl}, 10 \mathrm{mM}$ EDTA, $0.5 \%$ sarcosyl, and $1 \mathrm{mg} / \mathrm{mL}$ proteinase $\mathrm{K}$ ) and incubated overnight at $60^{\circ} \mathrm{C}$. Genomic DNA was extracted from lysates by standard phenol/ chloroform technique and subjected to bisulfite conversion using EpiTect bisulfite kit (Qiagen) according to the manufacturer's protocols. DNA was quantified and then diluted to a working concentration of $20 \mathrm{ng} / \mu \mathrm{L}$ for BiSulfite Amplicon Sequencing (BSAS) [48]. CpG islands located in the proximal promoter of AVPR1a, OXTR, and $P K C B$ were selected according to the following criteria: (1) $\geq 200$ bp length, (2) $\geq 50 \%$ GC content, (3) $\geq$ $60 \%$ ratio of observed/expected dinucleotides CpG. Based on the genomic coordinates of the candidate CpG sites (Table 3, 4, 8), we carefully designed the BSAS primers in order to detect them in a panel (Table 7). After PCR amplification, products were sequenced by Illumina Hiseq 2000. Methylation level at each tested CpG site was calculated as the percentage of the methylated cytosines over the total tested cytosines. The average methylation levels were calculated using methylation 
Table 7 List of oligonucleotide primers used in this study

\begin{tabular}{|c|c|c|}
\hline \multirow[t]{2}{*}{ Primer } & \multicolumn{2}{|l|}{ Nucleotide Sequence (5' to 3') } \\
\hline & Sense & Anti-sense \\
\hline \multicolumn{3}{|c|}{ qRT-PCR primers } \\
\hline AVPR1a & TCGTGACGGCTTACATCGTC & GAGTCTTGAAGGAGATGGCCA \\
\hline AVPR1b & CCTCATCTGCCATGAGATCTG & GCCACATTGGTGGAATCTTCATCA \\
\hline AVPR2 & TGACGCTAGTGATTGTGGTC & GACACGCTGCTGCTGAAAGA \\
\hline OTXR & TCAGCAGCGTCAAGCTCATC & GTGAACAGCATGTAGATCCAG \\
\hline PKCa & CTCTGCGGAATGGATCACACT & GGACTCATTCCACTGCGGAT \\
\hline PKC $\beta$ & GACCTCATGTATCACATCCAG & GAGTGCCACAGAATGTCTTG \\
\hline PKC & TCCAAGGACATCCTGGAGAAG & GTCTCTGGGTGACTTCACTT \\
\hline PKC $\varepsilon$ & TACAAGGTCCCTACCTTCTG & TCGGCCAGTACTITGGCGAT \\
\hline PKCY & TGCCTGTGCCCGTCATATCCT & AGAGTCCAGAACGCTAAGGT \\
\hline \multicolumn{3}{|c|}{ Bisulfite sequencing primers } \\
\hline AVPR1a & AGAGTTAGGTTTAGGTGTAGGAGTTAGATG & CAAATTCCTCCTCACAATAAATAAAATC \\
\hline OTXR & TTYYGTTYGGAGGGGTTTG & AATACTAAACTAAAATCTCTCACTAAAACCTC \\
\hline PKC $\beta-1$ & GGTAGTAGTTGGGYGAGTGATAgttt & ACCCCRCAACCRAATCAAC \\
\hline PKC $\beta-2$ & GgtttYGgggtYGgtATTाT & CTCACCAAATAAAATCRATACAATAACTACAAA \\
\hline
\end{tabular}

Table 8 Methylated CpG sites at PKCB gene promoter measured in this study

\begin{tabular}{|c|c|c|c|c|c|c|}
\hline Gene & Position & Genomic location & Relative to TSS, bp & Position & Genomic location & Relative to TSS, bp \\
\hline \multirow[t]{22}{*}{$P K C B$} & 1 & Chr16: 23847338 & +27 & 23 & Chr16: 23847448 & 137 \\
\hline & 2 & Chr16: 23847344 & +33 & 24 & Chr16: 23847450 & 139 \\
\hline & 3 & Chr16: 23847346 & +35 & 25 & Chr16: 23847456 & 145 \\
\hline & 4 & Chr16: 23847348 & +37 & 26 & Chr16: 23847462 & 151 \\
\hline & 5 & Chr16: 23847351 & +40 & 27 & Chr16: 23847472 & 157 \\
\hline & 6 & Chr16: 23847353 & +42 & 28 & Chr16: 23847479 & 164 \\
\hline & 7 & Chr16: 23847357 & +46 & 29 & Chr16: 23847487 & 172 \\
\hline & 8 & Chr16: 23847366 & +55 & 30 & Chr16: 23847489 & 174 \\
\hline & 9 & Chr16: 23847369 & +58 & 31 & Chr16: 23847491 & 176 \\
\hline & 10 & Chr16: 23847380 & +69 & 32 & Chr16: 23847507 & 192 \\
\hline & 11 & Chr16: 23847384 & +73 & 33 & Chr16: 23847513 & 198 \\
\hline & 12 & Chr16: 23847386 & +75 & 34 & Chr16: 23847519 & 204 \\
\hline & 13 & Chr16: 23847392 & +81 & 35 & Chr16: 23847522 & 207 \\
\hline & 14 & Chr16: 23847404 & +93 & 36 & Chr16: 23847525 & 210 \\
\hline & 15 & Chr16: 23847408 & +97 & 37 & Chr16: 23847529 & 214 \\
\hline & 16 & Chr16: 23847411 & +100 & 38 & Chr16: 23847535 & 220 \\
\hline & 17 & Chr16: 23847418 & +107 & 39 & Chr16: 23847547 & 232 \\
\hline & 18 & Chr16: 23847420 & +109 & 40 & Chr16: 23847551 & 236 \\
\hline & 19 & Chr16: 23847428 & +117 & 41 & Chr16: 23847556 & 241 \\
\hline & 20 & Chr16: 23847433 & +122 & 42 & Chr16: 23847560 & 245 \\
\hline & 21 & Chr16: 23847440 & +129 & 43 & Chr16: 23847568 & 253 \\
\hline & 22 & Chr16: 23847442 & +131 & 44 & Chr16: 23847575 & 260 \\
\hline
\end{tabular}


levels of all measured CpG sites within the AVPR1a, $O X T R$, or $P K C B$ gene.

\section{Isolation and culture HUVECs}

Umbilical cords (about $15 \mathrm{~cm}$ in length) were excised from the placenta immediately after delivery and placed into cold sterile phosphate-buffered saline. Endothelial cells were isolated from umbilical veins as described previously [46]. HUVECs were cultured in DMEM containing $20 \%$ fetal bovine serum at $37^{\circ} \mathrm{C}$ with $5 \% \mathrm{CO}_{2}$ and $95 \%$ air humidified incubator. Cultures were passaged every $2-3$ days and used in experiments 2 passages. In 5-Aza treatment studies, HUVECs were seeded and allowed to grow for 2 days with or without 5-Aza (Sigma-Aldrich, $10^{-6} \mathrm{~mol} / \mathrm{L}$ ) and then mRNA were extracted for experiments.

\section{Data analysis and statistics}

All data were expressed as the mean \pm SEM. Significance $(P<0.05)$ was ascertained by $t$ test or two-way analysis of variance (ANOVA) followed by the Bonferroni test. Concentration-dependent response curves were performed by computer-assisted nonlinear regression. DNA methylation/mRNA correlation plots were identified by causal inference test (Graph Pad Prism software CA, USA).

\section{Abbreviations}

Atosiban: OXT-specific antagonist; AVP: Arginine vasopressin; GF109203X: PKC antagonist; HUV: Human umbilical vein; KCl: Potassium chloride; NP: Normal pregnancies; OXT: Oxytocin; PDBu: Phorbol 12, 13-dibutyrate (PKC activator) PE: Pre-eclampsia; PKC: Protein kinase C; SR49059: AVPR1a-specific antagonist

\section{Acknowledgments}

We thank Tian Hao Biotechnology Co., Ltd., Shanghai, China for the excellent technical assistance.

\section{Authors' contributions}

QG processed the data and figures and performed vessel experiments with FX, HL, TX, HD, YY, and YH. QG, FX, and TX performed the molecular studies. JC, JT, and ZX prepared the human umbilical cord samples. The work was supervised by ZX and QG. All authors read and approved the final manuscript.

\section{Funding}

Supported partly by the National Nature \& Science Foundation of China (81873841, 81741024, 81401244, 81771592, and 81320108006), the Natural Science Foundation of Jiangsu Province (BK20140292), "333 Project," "Six one project (LGY2018076)," "Shuang Chuang Tuan Dui" and Key Discipline "Fetal medicine" of Jiangsu Province, and the Suzhou city "Wei Sheng Ren Cai (GSWS2019029)" program.

\section{Ethics approval and consent to participate}

The studies were approved by the institutional review boards of the First Hospital of Soochow University at Jiangsu Province, China. Written informed consent was obtained from each study subject.

\section{Consent for publication}

Not applicable

\section{Competing interests}

The authors declare that they have no competing interests.

\section{Author details}

'Institute for Fetology and Department of Obstetrics and Gynecology, First Hospital of Soochow University, Suzhou 215006, China. ${ }^{2}$ Center for Perinatal Biology, Loma Linda University, California, USA. ${ }^{3}$ Department of Obstetrics and Gynecology, Suzhou Municipal Hospital, Suzhou, China. ${ }^{4}$ Department of Obstetrics and Gynecology, Affiliated Suzhou Hospital of Nanjing University of Chinese Medicine, Suzhou, China.

Received: 20 February 2019 Accepted: 15 May 2019

Published online: 28 May 2019

\section{References}

1. Steegers EA, von Dadelszen P, Duvekot JJ, Pijnenborg R. Pre-eclampsia. Lancet. 2010;376:631-44.

2. Chaiworapongsa T, Chaemsaithong P, Yeo L, Romero R. Pre-eclampsia part 1: Current understanding of its pathophysiology. Nat Rev Nephrol. 2014;10: 466-80.

3. Walker DW, McLean JR. Absence of adrenergic nerves in the human placenta. Nature. 1971;229:344-5.

4. Reilly FD, Russell PT. Neurohistochemical evidence supporting an absence of adrenergic and cholinergic innervation in the human placenta and umbilical cord. Anat Rec. 1977:188:277-86.

5. Japundzic-Zigon N. Vasopressin and oxytocin in control of the cardiovascular system. Curr Neuropharmacol. 2013;11:218-30.

6. Yang G, Li T, Xu J, Liu L. Pkc plays an important mediated effect in arginine vasopressin induced restoration of vascular responsiveness and calcium sensitization following hemorrhagic shock in rats. Eur J Pharmacol. 2010;628:148-54.

7. Koshimizu TA, Nakamura K, Egashira N, Hiroyama M, Nonoguchi H, Tanoue A. Vasopressin $v 1 \mathrm{a}$ and $\mathrm{v} 1 \mathrm{~b}$ receptors: from molecules to physiological systems. Physiol Rev. 2012;92:1813-64.

8. Koshimizu TA, Nasa Y, Tanoue A, Oikawa R, Kawahara Y, Kiyono Y, Adachi T, Tanaka T, Kuwaki T, Mori T, et al. V1a vasopressin receptors maintain normal blood pressure by regulating circulating blood volume and baroreflex sensitivity. Proc Natl Acad Sci U S A. 2006;103:7807-12.

9. Wellmann S, Benzing J, Cippa G, Admaty D, Creutzfeldt R, Mieth RA, Beinder E, Lapaire O, Morgenthaler NG, Haagen U, et al. High copeptin concentrations in umbilical cord blood after vaginal delivery and birth acidosis. J Clin Endocrinol Metab. 2010;95:5091-6.

10. Polin RA, Husain MK, James LS, Frantz AG. High vasopressin concentrations in human umbilical cord blood--lack of correlation with stress. J Perinat Med. 1977;5:114-9.

11. de Geest K, Thiery M, Piron-Possuyt G, Vanden Driessche R. Plasma oxytocin in human pregnancy and parturition. J Perinat Med. 1985;13:3-13.

12. Chard T, Hudson CN, Edwards CR, Boyd NR. Release of oxytocin and vasopressin by the human foetus during labour. Nature. 1971;234:352-4.

13. Arrowsmith S, Wray S. Oxytocin: Its mechanism of action and receptor signalling in the myometrium. J Neuroendocrinol. 2014;26:356-69.

14. Yang $G, X u J$, Li T, Ming J, Chen W, Liu L. Role of v1a receptor in avpinduced restoration of vascular hyporeactivity and its relationship to $\mathrm{mlcp}$ mlc20 phosphorylation pathway. J Surg Res. 2010;161:312-20.

15. Eto M, Kitazawa T, Brautigan DL. Phosphoprotein inhibitor cpi-17 specificity depends on allosteric regulation of protein phosphatase-1 by regulatory subunits. Proc Natl Acad Sci U S A. 2004;101:8888-93.

16. Ringvold $\mathrm{HC}$, Khalil RA. Protein kinase $\mathrm{c}$ as regulator of vascular smooth muscle function and potential target in vascular disorders. Adv Pharmacol. 2017;78:203-301.

17. Chen YL, Shepherd C, Spinelli W, Lai FM. Oxytocin and vasopressin constrict rat isolated uterine resistance arteries by activating vasopressin $\mathrm{v} 1 \mathrm{a}$ receptors. Eur J Pharmacol. 1999;376:45-51.

18. Okhovat M, Maguire SM, Phelps SM. Methylation of avpr1a in the cortex of wild prairie voles: effects of cpg position and polymorphism. R Soc Open Sci. 2017;4:160646.

19. Bodden C, van den Hove D, Lesch KP, Sachser N. Impact of varying social experiences during life history on behaviour, gene expression, and vasopressin receptor gene methylation in mice. Sci Rep. 2017;7:8719.

20. Okhovat M, Chen IC, Dehghani Z, Zheng DJ, Ikpatt JE, Momoh H, Phelps SM. Genetic variation in the developmental regulation of cortical avpr1a among prairie voles. Genes Brain Behav. 2018;17:36-48.

21. Smearman EL, Almli LM, Conneely KN, Brody GH, Sales JM, Bradley B, Ressler KJ Smith AK. Oxytocin receptor genetic and epigenetic variations: association with child abuse and adult psychiatric symptoms. Child Dev. 2016;87:122-34. 
22. Mamrut S, Harony H, Sood R, Shahar-Gold H, Gainer H, Shi YJ, BarkiHarrington L, Wagner S. DNA methylation of specific cpg sites in the promoter region regulates the transcription of the mouse oxytocin receptor. PLoS One. 2013;8:e56869.

23. Kusui C, Kimura T, Ogita K, Nakamura H, Matsumura Y, Koyama M, Azuma C, Murata Y. DNA methylation of the human oxytocin receptor gene promoter regulates tissue-specific gene suppression. Biochem Biophys Res Commun. 2001;289:681-6.

24. Hagiwara $K$, Ito $H$, Murate $T$, Miyata $Y$, Ohashi $H$, Nagai $H$. Prox1 overexpression inhibits protein kinase $\mathrm{c}$ beta ii transcription through promoter DNA methylation. Genes Chromosomes Cancer. 2012;51:1024-36.

25. Li C, Gao W, Gao Y, Yu C, Lv J, Lv R, Duan J, Sun Y, Guo X, et al. Age prediction of children and adolescents aged 6-17 years: an epigenome-wide analysis of DNA methylation. Aging. 2018;10:1015-26.

26. Liu S, Chen X, Chen R, Wang J, Zhu G, Jiang J, Wang H, Duan S, Huang J. Diagnostic role of wnt pathway gene promoter methylation in non small cell lung cancer. Oncotarget. 2017;8:36354-67.

27. Heo HJ, Tozour JN, Delahaye F, Zhao Y, Cui L, Barzilai N, Einstein FH. Advanced aging phenotype is revealed by epigenetic modifications in rat liver after in utero malnutrition. Aging Cell. 2016;15:964-72.

28. Yeung KR, Chiu CL, Pidsley R, Makris A, Hennessy A, Lind JM. DNA methylation profiles in preeclampsia and healthy control placentas. Am J Physiol Heart Circ Physiol. 2016;310:H1295-303.

29. Gao Q, Tang J, Chen J, Jiang L, Zhu X, Xu Z. Epigenetic code and potential epigenetic-based therapies against chronic diseases in developmental origins. Drug Discov Today. 2014;19:1744-50.

30. Murphy MO, Cohn DM, Loria AS. Developmental origins of cardiovascular disease: impact of early life stress in humans and rodents. Neurosci Biobehav Rev. 2017;74:453-65.

31. Buffington SA, Di Prisco GV, Auchtung TA, Ajami NJ, Petrosino JF, CostaMattioli M. Microbial reconstitution reverses maternal diet-induced social and synaptic deficits in offspring. Cell. 2016;165:1762-75.

32. Davis EF, Lazdam M, Lewandowski AJ, Worton SA, Kelly B, Kenworthy Y, Adwani S, Wilkinson AR, McCormick K, Sargent I, et al. Cardiovascular risk factors in children and young adults born to pre-eclamptic pregnancies: a systematic review. Pediatrics. 2012;129:e1552-61.

33. Alsnes IV, Vatten LJ, Fraser A, Bjorngaard JH, Rich-Edwards J, Romundstad PR, Asvold BO. Hypertension in pregnancy and offspring cardiovascular risk in young adulthood: prospective and sibling studies in the hunt study (nord-trondelag health study) in norway. Hypertension. 2017:69:591-8.

34. Beukers F, Aarnoudse-Moens CSH, van Weissenbruch MM, Ganzevoort W, van Goudoever JB, van Wassenaer-Leemhuis AG. Maternal psychological distress after severe pregnancy hypertension was associated with increased child behavioural problems at the age of 12. Acta Paediatrica. 2018.

35. Figueiro-Filho EA, Mak LE, Reynolds JN, Stroman PW, Smith GN, Forkert ND, Paolozza A, Ratsep MT, Croy BA. Neurological function in children born to pre-eclamptic and hypertensive mothers - a systematic review. Pregnancy Hypertens. 2017;10:1-6.

36. Lara E, Acurio J, Leon J, Penny J, Torres-Vergara P, Escudero C. Are the cognitive alterations present in children born from pre-eclamptic pregnancies the result of impaired angiogenesis? Focus on the potential role of the vegf family. Frontiers Physiology. 2018;9:1591.

37. Fields JA, Garovic VD, Mielke MM, Kantarci K, Jayachandran M, White WM, Butts AM, Graff-Radford J, Lahr BD, Bailey KR, Miller VM. Preeclampsia and cognitive impairment later in life. Am J Obstet Gynecol. 2017;217:74 e71-11.

38. Davis EF, Lewandowski AJ, Aye C, Williamson W, Boardman H, Huang RC, Mori TA, Newnham J, Beilin LJ, Leeson P. Clinical cardiovascular risk during young adulthood in offspring of hypertensive pregnancies: insights from a 20-year prospective follow-up birth cohort. BMJ Open. 2015;5:e008136

39. Bellamy L, Casas JP, Hingorani AD, Williams DJ. Pre-eclampsia and risk of cardiovascular disease and cancer in later life: systematic review and metaanalysis. Bmj. 2007;335:974

40. Kajantie E, Eriksson JG, Osmond C, Thornburg K, Barker DJ. Pre-eclampsia is associated with increased risk of stroke in the adult offspring: The helsinki birth cohort study. Stroke. 2009;40:1176-80.

41. Hakim J, Senterman MK, Hakim AM. Preeclampsia is a biomarker for vascular disease in both mother and child: the need for a medical alert system. Int J Pediatr. 2013;2013:953150.

42. Hoodbhoy Z, Hasan BS, Mohammed N, Chowdhury D. Impact of preeclampsia on the cardiovascular health of the offspring: a cohort study protocol. BMJ Open. 2018;8:e024331.
43. Gao Q, Tang J, Li N, Liu B, Zhang M, Sun M, Xu Z. What is precise pathophysiology in development of hypertension in pregnancy? Precision medicine requires precise physiology and pathophysiology. Drug Discov Today. 2018;23:286-99.

44. Young BC, Levine RJ, Karumanchi SA. Pathogenesis of preeclampsia. Annu Rev Pathol. 2010;5:173-92.

45. Zhu X, Gao Q, Tu Q, Zhong Y, Zhu D, Mao C, Xu Z. Prenatal hypoxia enhanced angiotensin ii-mediated vasoconstriction via increased oxidative signaling in fetal rats. Reprod Toxicol. 2016;60:21-8.

46. Gao Q, Zhu X, Chen J, Mao C, Zhang L, Xu Z. Upregulation of p53 promoted g1 arrest and apoptosis in human umbilical cord vein endothelia cells from preeclampsia. J Hypertens. 2016;34:1380-8.

47. Liu X, Gao Q, Li P, Zhao Q, Zhang J, Li J, Koseki H, Wong J. Uhrf1 targets dnmt1 for DNA methylation through cooperative binding of hemimethylated DNA and methylated h3k9. Nat Commun. 2013;4:1563.

48. Masser DR, Berg AS, Freeman WM. Focused, high accuracy 5-methylcytosine quantitation with base resolution by benchtop next-generation sequencing. Epigenetics Chromatin. 2013;6:33.

\section{Publisher's Note}

Springer Nature remains neutral with regard to jurisdictional claims in published maps and institutional affiliations.
Ready to submit your research? Choose BMC and benefit from:

- fast, convenient online submission

- thorough peer review by experienced researchers in your field

- rapid publication on acceptance

- support for research data, including large and complex data types

- gold Open Access which fosters wider collaboration and increased citations

- maximum visibility for your research: over $100 \mathrm{M}$ website views per year

At BMC, research is always in progress.

Learn more biomedcentral.com/submissions 